\title{
miRNA-320a inhibits glioma cell invasion and migration by directly targeting aquaporin 4
}

\author{
WEI XIONG ${ }^{1,2}$, JIANHUA RAN $^{3}$, RONG JIANG $^{1}$, PEI GUO $^{3}$, XUEPING SHI $^{1}$, \\ HAIXING LI ${ }^{1}$, XIAOTING LV ${ }^{1}$, JING LI ${ }^{1}$ and DILONG CHEN ${ }^{1,2}$ \\ ${ }^{1}$ Laboratory of Stem Cell and Tissue Engineering, Chongqing Medical University, Chongqing 400016; \\ ${ }^{2}$ Chongqing Three Gorges Medical College, Chongqing 404120; ${ }^{3}$ Institute of Neuroscience, \\ Chongqing Medical University, Chongqing 400016, P.R. China
}

Received August 3, 2017; Accepted February 7, 2018

DOI: $10.3892 /$ or.2018.6274

\begin{abstract}
Glioma is the most aggressive and malignant type of primary intracranial tumor. In recent decades, despite the rapid development of modern surgery and therapeutic strategies available for brain tumors, the prognosis of glioma remains poor and the median survival time is $<15$ months. In this study, we found that the levels of miRNA-320a were significantly decreased in patients with glioma, and that elevated miRNA-320a expression was associated with a better prognosis. In addition, aquaporin 4 (AQP4) was identified as a direct target of miRNA-320a. Overexpression of miRNA-320a led to the inhibition of cell invasion and migration via targeting of AQP4. Therefore, our results suggested that miRNA-320a could suppress the aggressive capacity of tumors by targeting AQP4, and that miRNA-320a could serve as a new effective therapeutic target for glioma surgical and therapeutic strategies.
\end{abstract}

\section{Introduction}

In the central nervous system, gliomas are the most common and malignant types of tumor. The WHO tumor grades are significantly associated with patient prognosis (1). Due to the tumor invasiveness and difficulty in identifying tumor boundaries, the prognosis remains poor even with use of the current standard therapies. Scientists have previously indicated that tumor microenvironmental changes and gene mutations [including microRNAs (miRNAs) and long non-coding RNAs (lncRNAs)] each control glioma progression and prognosis (2-5). Although modern healthcare has developed rapidly,

Correspondence to: Dr Jing Li or Professor Dilong Chen, Laboratory of Stem Cell and Tissue Engineering, Chongqing Medical University, 1 Yixueyuan Road, Yuzhong, Chongqing 400016, P.R. China E-mail: lijingyangyang@126.com

E-mail: chendilong@21cn.com

Key words: glioma, miRNA-320a, aquaporin 4, invasion, migration especially in the application of individualized treatments $(6,7)$, effective and accessible novel therapeutic strategies are urgently required to combat this malignancy.

miRNAs are a class of small non-coding RNAs that negatively regulate their targets by binding to the 3'-untranslated regions (3'-UTRs) of the mRNA, and then degrade their target mRNA or hinder the expression of their target protein (8). Studies have shown that miRNAs regulate various physiological and pathological processes, such as cell division, differentiation and death, as well as cancer progression, stem-cell differentiation and self-renewal $(4,9,10)$. In recent years, miRNAs have been widely studied and noted as biomarkers (11). miRNA genes are generally located at fragile chromosomal sites, and increasing evidence suggests that miRNAs serve an important role in cancer progression (12). Various human cancer types are accompanied by aberrant expression of miRNAs, which can function as tumor suppressors or oncogenes. Recently, miRNA-320a has been reported to regulate cell proliferation, differentiation and invasion in human colon cancer (13), and erythroid differentiation (14) as well as bladder carcinoma (15). However, there are few studies demonstrating an association between miRNA-320a and glioma.

Aquaporin 4 (AQP4) is a protein of the aquaporin family, which is distributed in different areas of the central nervous system (16). AQP4 is the AQP subtype abundantly expressed on astrocytes, and serves an important role in fluid exchange between the cerebrospinal fluid compartments and the brain $(17,18)$. Recently, AQP4 regulation has been reported to have a role in various brain diseases, including cerebral edema (19), Alzheimer's disease (20) and gliomas (21,22). It not only controls water exchange, but can also regulate the expression of amyloid- $\beta$ peptides $(\mathrm{A} \beta)$, and influence $\mathrm{K}^{+}$and $\mathrm{Ca}^{2+}$ transport (20). Numerous studies have suggested the importance of AQP4 in the physiological and pathological processes of the brain. In cancer research, AQP4 has also been associated with cell apoptosis and adhesion (23,24); thus, AQP4 may be a target for certain cancer therapies.

AQP4 is highly expressed at perivascular astrocyte end-feet, influencing cell membrane dissociation and recombination. In addition, it is highly expressed in gliomas and contributes to tumor progression $(17,25,26)$. Therefore, we hypothesized that AQP4 could regulate cell invasion and migration in glioma. 
In this study, we obtained further knowledge regarding the associations between AQP4 and miRNA-320a. We found that miRNA-320a was downregulated in glioma tissues compared to normal tissues, and we identified AQP4 to be a direct target of miRNA-320a that mediates glioma cell invasion and migration.

\section{Materials and methods}

CGGA data analyses and miRNA target prediction. miRNA-320a expression values and associated prognostic information from 198 glioma cases were obtained from the Chinese Glioma Genome Atlas (CGGA; http://www.cgga.org. $\mathrm{cn})$. These 198 samples were comprised of $135 \mathrm{WHO}$ III and IV tumors, and 63 WHO II tumors. The Kaplan-Meier estimation method was used for overall survival analysis of patients based on miRNA expression. Candidate targets of miRNA-320a were predicted by miRBase (http://mirtarbase.mbc.nctu.edu. tw/index.php), TargetScan (http://www.targetscan.org/vert_71) and miRanda (http://www.microrna.org/microrna/home.do).

Cell culture and human tissue samples. The U87 and U251 human glioma cell lines were cultured in DMEM (HyClone; GE Healthcare Life Sciences, Logan, UT, USA) supplemented with $10 \%$ fetal bovine serum (FBS; Gemini Bio Products, West Sacramento, CA, USA), $100 \mathrm{U} / \mathrm{ml}$ penicillin and $100 \mathrm{ng} / \mathrm{ml}$ streptomycin (both from Beyotime Institute of Biotechnology, Haimen, China). All cells were incubated at $37^{\circ} \mathrm{C}$ in an atmosphere containing $5 \% \mathrm{CO}_{2}$. Human glioma and non-cancerous human brain paraffin-embedded tissues were obtained from the Institute of Neuroscience Ran Jianhua research group. These tissue samples were subsequently used for immunohistochemistry (IHC) and for hematoxylin and eosin (H\&E) staining.

miRNA mimic and siRNA transfection. Hsa-miRNA-320a, miRNA-negative control (NC) mimics and AQP4 siRNA were chemically synthesized by RiboBio Co., Ltd. (Guangzhou, China). Transfection of hsa-miRNA-320a, miRNA-NC or siRNA was performed using the riboFECT ${ }^{\mathrm{TM}} \mathrm{CP}$ reagent (RiboBio Co., Ltd.), according to the manufacturer's instructions. miRNA-320a or siRNA (Table I) was transfected into the U87 and U251 glioma cells for $48 \mathrm{~h}$, prior to further experiments being performed.

Dual-luciferase reporter assay. The 3'-UTR of AQP4 contains three predicted matching regions for miRNA-320a. For the luciferase reporter assay, Luci-AQP4 and the NC were designed and synthesized by Gene Create Biological Engineering Co., Ltd. (Wuhan, China). First, 293T cells were transfected with an AQP4-3'-UTR-luciferase plasmid, followed by transfection with the miRNA-320a mimic or miRNA-NC in 48-well plates. The cells were then collected and lysed for a luciferase assay $48 \mathrm{~h}$ after transfection. Renilla luciferase was used for normalization.

RNA isolation and reverse transcription-quantitative PCR (RT-qPCR) analysis. The total RNAs were extracted from U87 and U251 cells using a HiPure Universal miRNA kit (Magen, Guangzhou, China). The RT-qPCR analysis was performed using an All-in-One miRNA RT-qPCR Detection kit
Table I. The interference sequences of AQP4.

SiRNAs Sequences

$\begin{array}{ll}\text { AQP4 siRNA1 } & \text { CCAAGTCTGTCTTCTACAT } \\ \text { AQP4 siRNA2 } & \text { GTTGAATTCAAACGTCGTT } \\ \text { AQP4 siRNA3 } & \text { TTTACCGGTCGACATGGTT }\end{array}$

AQP4, aquaporin 4.

(GeneCopoeia, Inc., Rockville, MD, USA), as previously described (27), with a T100 ${ }^{\mathrm{TM}}$ Thermal Cycler and a CFX Connect $^{\mathrm{TM}}$ Real-Time System (Bio-Rad Laboratories, Inc., Hercules, CA, USA), according to the manufacturer's instructions. The associated expression values of miRNA-320a were calculated using a comparative method following normalization to U6 rRNA. The specific primers were all designed and synthesized by GeneCopoeia, Inc. (mature has-miRNA-320a: 5'-AAAAGCUGGGUUGAGAGGGCGA-3'). The mRNA was extracted using RNAiso plus, and converted to cDNA using a PrimeScript $^{\mathrm{TM}}$ RT Reagent kit (both from Takara Bio, Inc., Otsu, Japan). RT-qPCR was performed using SYBR Premix Ex Taq II (Takara Bio, Inc.). SYBR-Green primer sequences were as follows: AQP4 forward, 5'-TCAGCATCGCCAAG TCTGTC-3' and reverse, 5'-CTGGGAGGTGTGACCAGA TAG-3'; MMP9 forward, 5'-CCCGGACCAAGGATAC AGT-3' and reverse, 5'-GCCATTCACGTCGTCCTTA-3'; $\beta$-actin forward, 5'-ACTGGGACGACATGGAAAAG-3' and reverse, 5'-TACATGGCTGGGACATTGAA-3'.

Invasion and migration assays. A Transwell chamber assay was used to assess cell invasion and migration, according to the manufacturer's instructions. For the invasion assay, $2 \times 10^{4}$ transfected cells were seeded into the upper chambers, which were coated with extracellular matrix (ECM) (BD Biosciences, San Jose, CA, USA). The migration assay did not use ECM-coated chambers (Costar; Corning Incorporated, Corning, NY, USA). After incubation at $37^{\circ} \mathrm{C}$ for $24 \mathrm{~h}$, the cells that were adherent to the upper surface of the filter were removed, and the migrated cells on the lower surfaces were fixed and then stained with crystal violet (Beyotime Institute of Biotechnology).

IHC and western blotting. IHC and western blot analyses were performed as previously described (28). Primary antibodies included the following: Mouse mAb AQP4 (1:300; cat. no. sc-32739) for IHC (Santa Cruz Biotechnology, Inc., Santa Cruz, CA, USA), rabbit mAb AQP4 for western blotting $(1: 1,000$; cat. no. BS3436) and rabbit $\mathrm{mAb} \beta$-actin $(1: 10,000$; cat. no. AP0060) (both from Bioworld Technology, Inc., St. Louis Park, MN, USA), and a rabbit mAb MMP9 (1:800; cat. no. w101580) (Wanleibio Co., Ltd., Shenyang, China). A secondary anti-rabbit antibody (1:10,000; cat. no. BS1043) (Bioworld Technology, Inc.) was used, as well as a biotin-streptavidin HRP detection system (ZSGB-Bio, Beijing, China). The western blot analyses were visualized via chemiluminescence reagents (EMD Millipore, Billerica, MA, USA) and a Bio-Rad ChemiDoc ${ }^{\mathrm{TM}}$ Touch Imaging System (Bio-Rad 
A

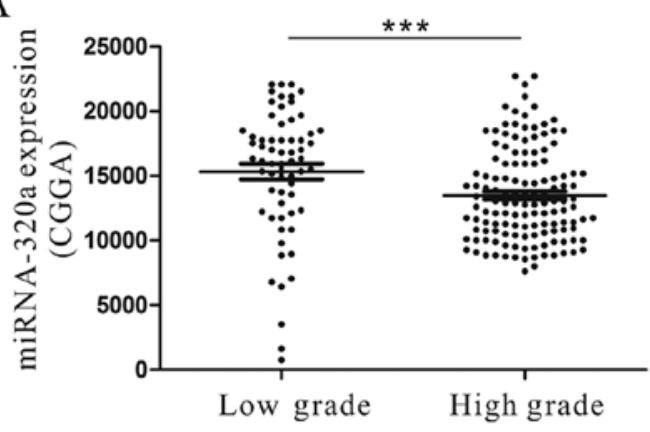

B

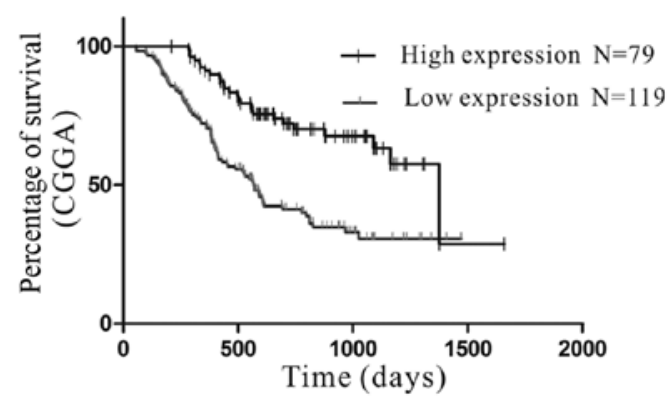

Figure 1. The expression of miRNA-320a in glioma tissues and the prognosis of patients from the CGGA. (A) High grade (III and IV) vs. low grade (II) ( $\left.{ }^{* * *} \mathrm{P}<0.001\right)$. (B) Kaplan-Meier curve of the glioma clinical outcome for miRNA-320a from the CGGA (P<0.01; 198 patients, log-rank test). CGGA, Chinese Glioma Genome Atlas.

Laboratories, Inc.). IHC staining was visualized using a light microscope (Olympus Corporation, Tokyo, Japan) and MicroPublisher v.5.0 RTV software (QImaging, Surrey, BC, Canada).

$H \& E$ staining. The paraffin-embedded tissue sections were sliced into 4- $\mu$ m-thick sections, deparaffinized in dimethylbenzene, and then rehydrated in $100 \%$ and $80 \%$ alcohol and distilled water. Subsequently, H\&E was used to stain the tissue sections, which were then imaged under a light microscope (Olympus Corporation) and analyzed with MicroPublisher v.5.0 RTV software.

Immunofluorescence staining. In brief, the U87 and U251 glioma cells were transfected with miRNA-320a or miRNA-NC and AQP4-siRNA or NC at $37^{\circ} \mathrm{C}$ with $5 \% \mathrm{CO}_{2}$ for $48 \mathrm{~h}$. The cells were then washed three times with ice-cold PBS and fixed in $4 \%$ paraformaldehyde. Normal goat serum (HyClone; GE Healthcare Life Sciences) was used to block the cell membranes for $30 \mathrm{~min}$ at room temperature, then the primary mouse mAb AQP4 (1:1,000; cat. no. sc-32739) was added and incubated overnight at $4^{\circ} \mathrm{C}$. The anti-mouse secondary fluorescent antibody (1:64; cat. no. BA1101) (Boster Biological Technology, Ltd., Wuhan, China) was subsequently added and incubated for $1 \mathrm{~h}$ at $37^{\circ} \mathrm{C}$, followed by staining with PI (Beyotime Institute of Biotechnology). Following this, 50\% glycerol was used for mounting, and images were obtained via fluorescence microscopy (Olympus Corporation).

Statistical analysis. The data were analyzed using SPSS v.20.0 (IBM SPSS, Chicago, IL, USA) and the results from each transfected group were compared against the NC group using a t-test. $\mathrm{P}<0.05$ was considered to indicate a statistically significant difference.

\section{Results}

miRNA-320a is downregulated in glioma tissues and is associated with patient prognosis. miRNA-320a has been reported to be downregulated in chronic myeloid leukemia and non-small cell lung cancer $(29,30)$. To investigate the expression of miRNA-320a in glioma, microarray data from

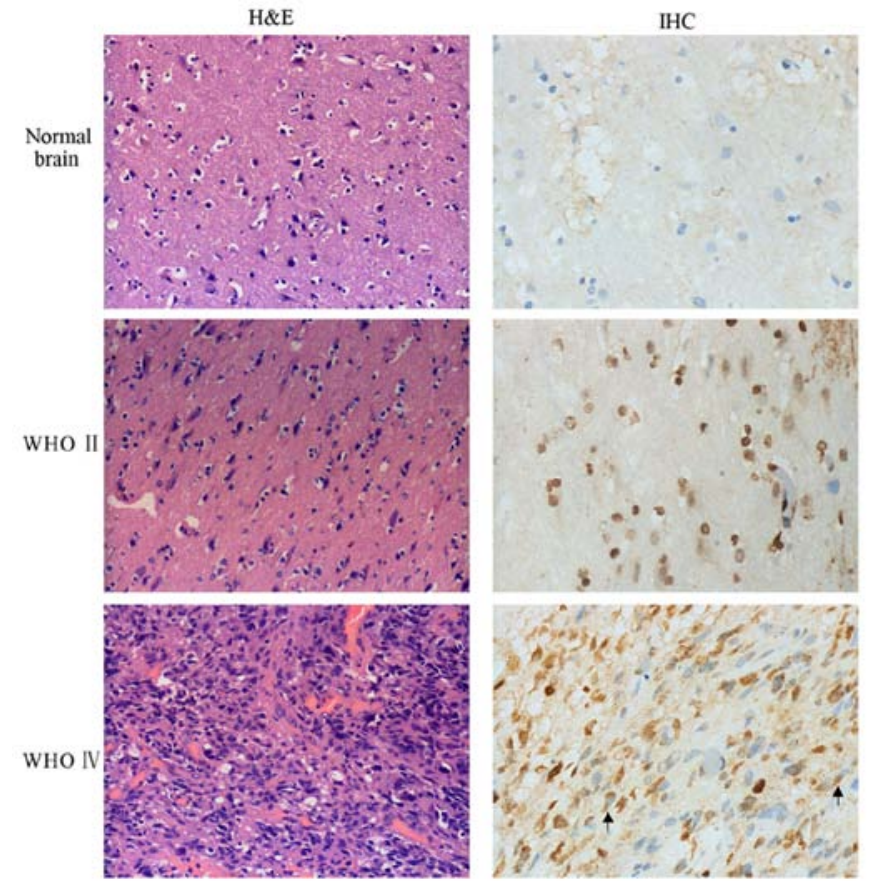

Figure 2. Normal tissue and various tumor tissues in the human brain were stained with H\&E, and the expression of AQP4 in tissues was determined by IHC. H\&E, hematoxylin and eosin; AQP4, aquaporin 4.

the CGGA were analyzed. The microarray data and clinical information from the CGGA included a total of 198 high-grade and low-grade glioma samples. The results indicated that the miRNA-320a expression was significantly lower in high-grade (grade III-IV) glioma samples compared with in low-grade (grade II) glioma samples ( $\mathrm{P}<0.01$; Fig. 1A). In addition, the miRNA-320a expression in grade IV glioma tissues was significantly different compared with that in grade II glioma and normal brain tissues. Furthermore, in human glioma cell lines, miRNA-320a was significantly decreased compared with that in human astrocytes (31).

We identified that the expression of miRNA-320a was associated with overall survival in each of the 198 glioma samples from the CGGA. We determined that high miRNA-320a expression was significantly associated with a better prognosis $(\mathrm{P}<0.01$; Fig. 1B). The results demonstrated that miRNA-320a expression was downregulated in glioma tissues and cell lines, 
A

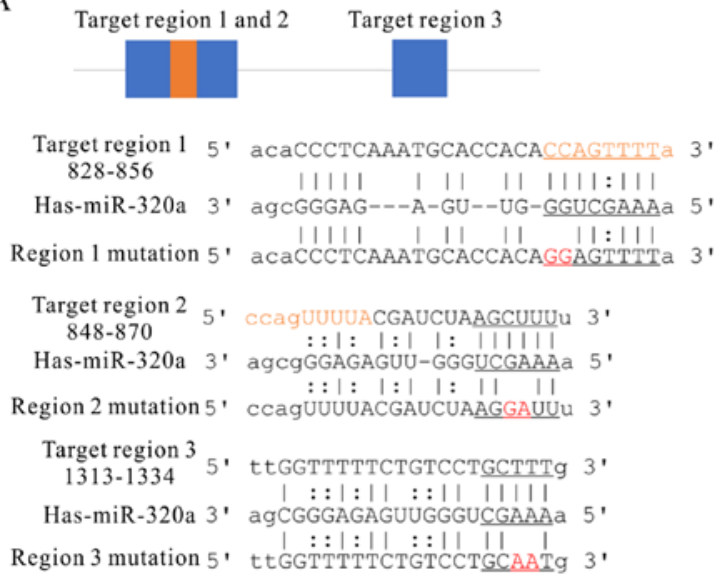

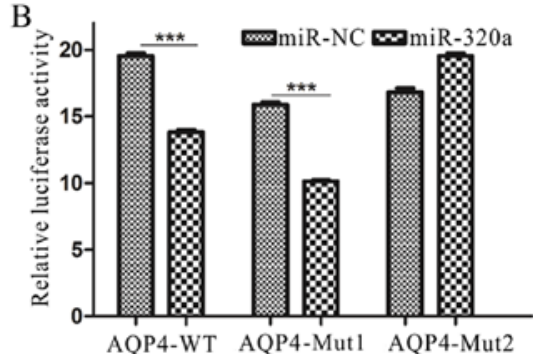

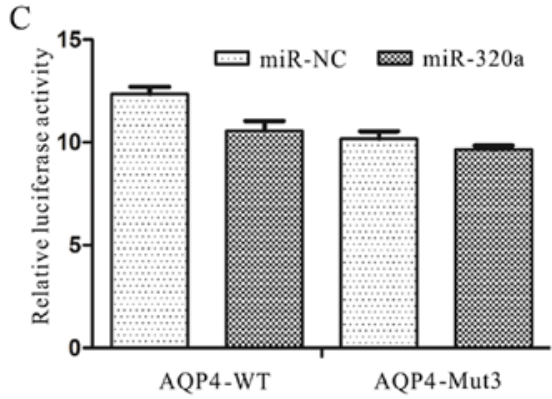

Figure 3. AQP4 is a direct target of miR-320a. (A) Bioinformatics analysis of the predicted interactions of miR-320a and its binding sites within the 3'-UTR of AQP4. Target regions 1 and 2 are very close in the 3 '-UTR, the orange region is the overlapping ribonucleic acid, thus region 3 was identified by an independent wild or mutation plasmid. The underlined sequence indicates the target region. (B and C) The luciferase activity of the cells was identified after transfection for $48 \mathrm{~h}$ (normalized to Renilla luciferase activity, $\left.{ }^{* * *} \mathrm{P}<0.001\right)$. AQP4, aquaporin 4.
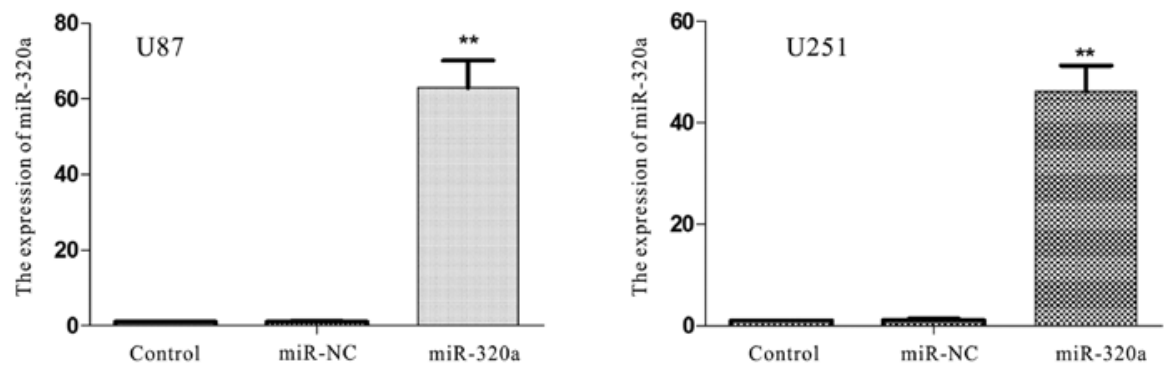

Figure 4. The expression of miRNA-320a in glioma cells. Overexpression of miRNA-320a in U87 and U251 cell lines after transfection with miRNA-320a and NC mimics (normalized to U6; vs. miR-NC, ${ }^{* *} \mathrm{P}<0.01$ )

and that high miRNA-320a expression was associated with an improved patient prognosis, consistent with prior studies (32). Therefore, the data revealed that miRNA-320a may function as a tumor suppressor in glioma.

AQP4 is upregulated in human glioma tissue samples. IHC was used to investigate the expression of AQP4 in human glioma and normal brain tissues (Fig. 2). Compared with the WHO grade II and normal brain tissues, H\&E staining clearly revealed cellular atypia and an abundance of tumor cells in the WHO grade IV glioma samples. The nuclei were nonuniform in size, and exhibited hyperchromatism and nuclear division. Regarding AQP4 expression, the high-grade glioma tissues almost express AQP4 in whole cells, and showed a polarized distribution. In grade II glioma tissues, few cells expressed AQP4 in the nucleus. By comparison, in normal brain tissues, AQP4 was expressed only in perivascular cells. Prior studies indicated that downregulated AQP4 could suppress cell proliferation and motility (33). Those results demonstrated that the expression of AQP4 was significantly correlated with the glioma WHO grade, and had a key role in the cancer progression.
AQP4 is a direct target of miRNA-320a. To demonstrate the mechanism of miRNA-320a in glioma, we searched for miRNA-320a targets using the algorithms TargetScan, miRanda and miRbase. AQP4 was selected from several putative miRNA-320a targets since it plays key roles in brain diseases, cell adhesion and motility $(16,19,24,33)$. Luciferase assays were conducted to examine whether AQP4 was a direct target of miRNA-320a. As shown in Fig. 3, three 3'-UTR regions of AQP4 mRNA were complementary to miRNA-320a. The results indicated that miRNA-320a could directly target a region within the 2,000 bp of the AQP4 3'-UTR (Fig. 3A). Therefore, using the luciferase reporter system, we ascertained that the overexpression of miRNA-320a in U87 and U251 cells suppressed the activity of AQP4 $(\mathrm{P}<0.01$; Fig. 3B and $\mathrm{C})$.

UpregulationmiRNA-320ainhibits cell invasionandmigration. To further explore the biological functions of miRNA-320a in glioma cell lines, U87 and U251 cells were transfected with miRNA-320a or miRNA-NC mimics. RT-qPCR revealed that miRNA-320a expression was significantly increased in the transfected U87 and U251 cells, compared with that in the control and miR-NC groups $(\mathrm{P}<0.01$; Fig. 4). A Transwell 

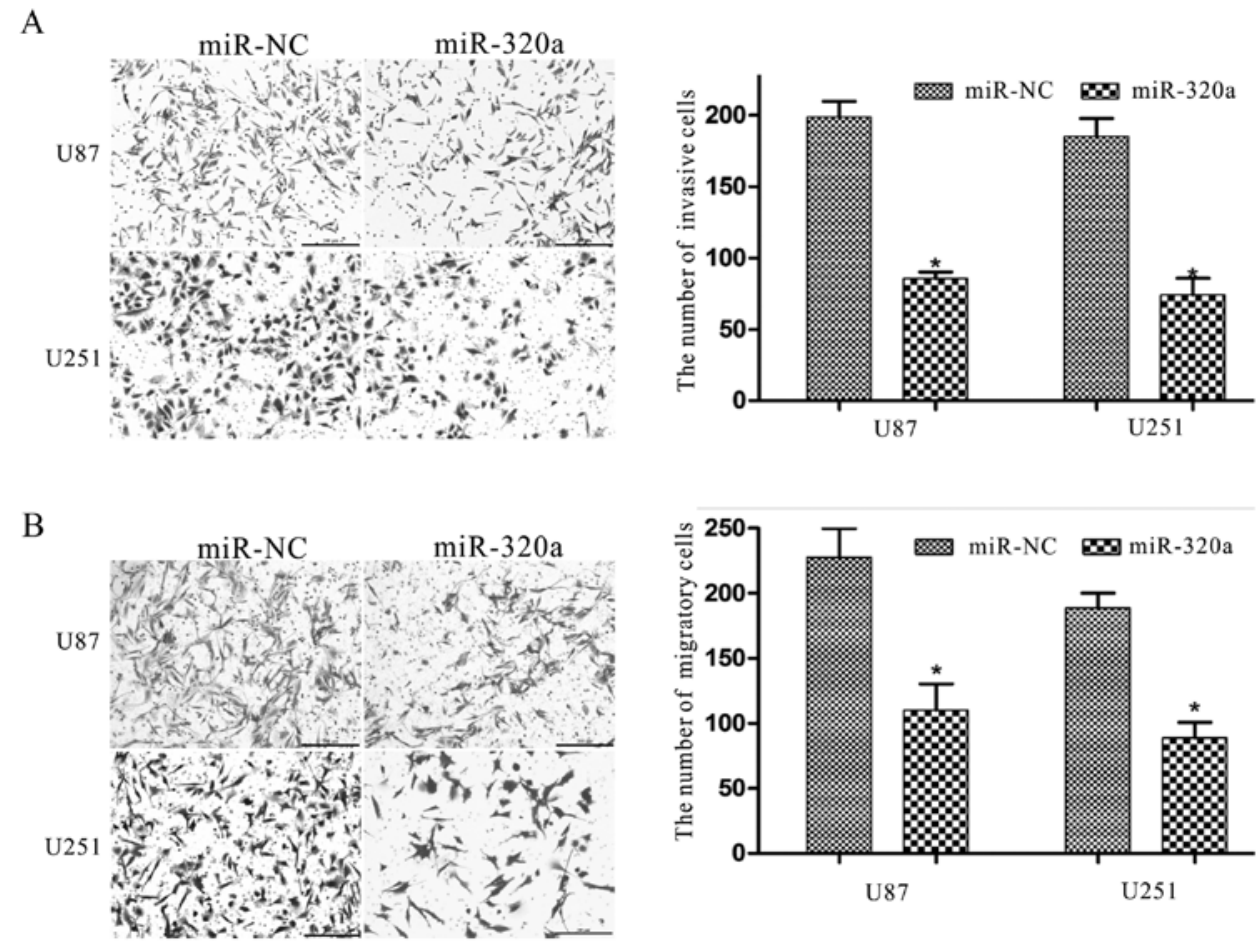

Figure 5. Cell invasion and migration are inhibited by overexpression of miR-320a. (A) The Transwell inner chamber was coated with matrix gel for cell invasion detection and cell counting. (B) The Transwell chamber without matrix gel directly determined the cell migration. ("P<0.05).
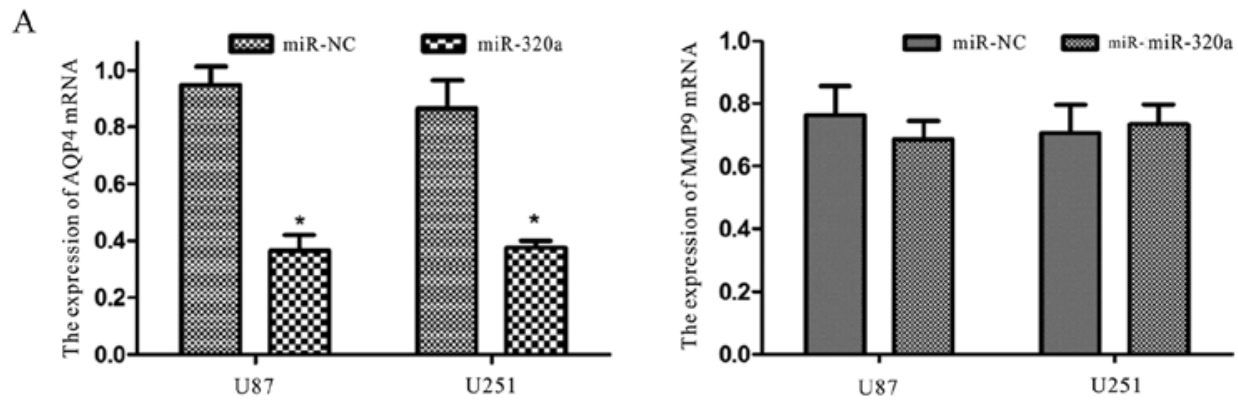

B
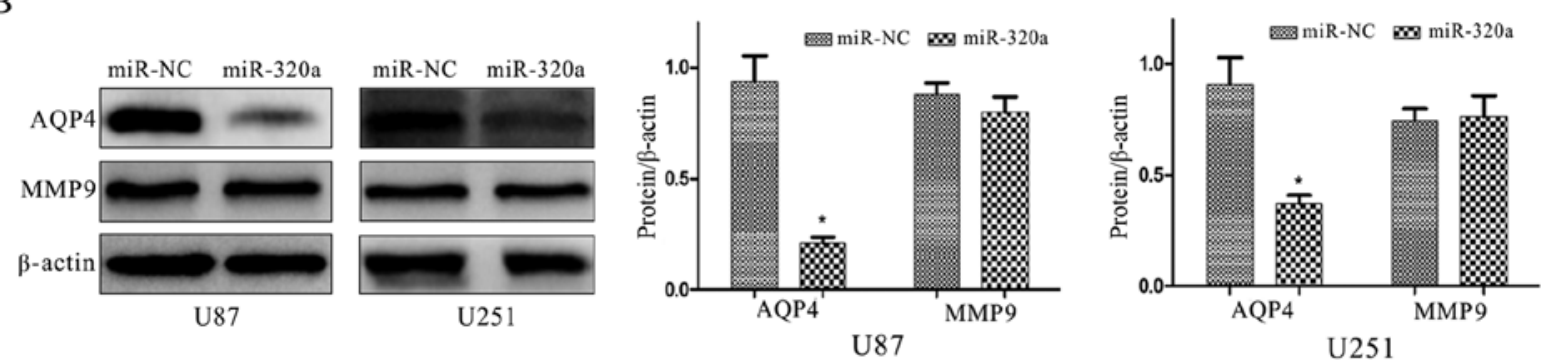

Figure 6. The level of AQP4 was downregulated by overexpression of miR-320a. (A) AQP4 and MMP9 mRNA expression determined by RT-PCR. (B) The expression of AQP4 and MMP9 proteins were identified by western blot analysis ( $\mathrm{P}<0.05)$. AQP4, aquaporin 4.

assay was conducted to determine the migratory and invasive behavior of U87 and U251 cells, and was used to detect the function of miRNA-320a in the progression of glioma. We found that miRNA-320a overexpression significantly decreased the migratory and invasive capacities of U87 and U251 cells (Fig. 5A and B). In addition, miRNA-320a has been demonstrated to suppress the proliferation of K-562 chronic myelogenous leukemia, LN-229 glioblastoma and U2OS osteosarcoma cells $(29,31,34)$. The results of these prior studies have each demonstrated that miRNA-320a could function as a tumor suppressor, and regulate progression and motility in glioma cells, as well as in other types of cancer.

Upregulation of miRNA-320a suppresses the expression of AQP4. Luciferase assays were conducted using U87 and U251 cells in order to identify changes in miRNA-320a 


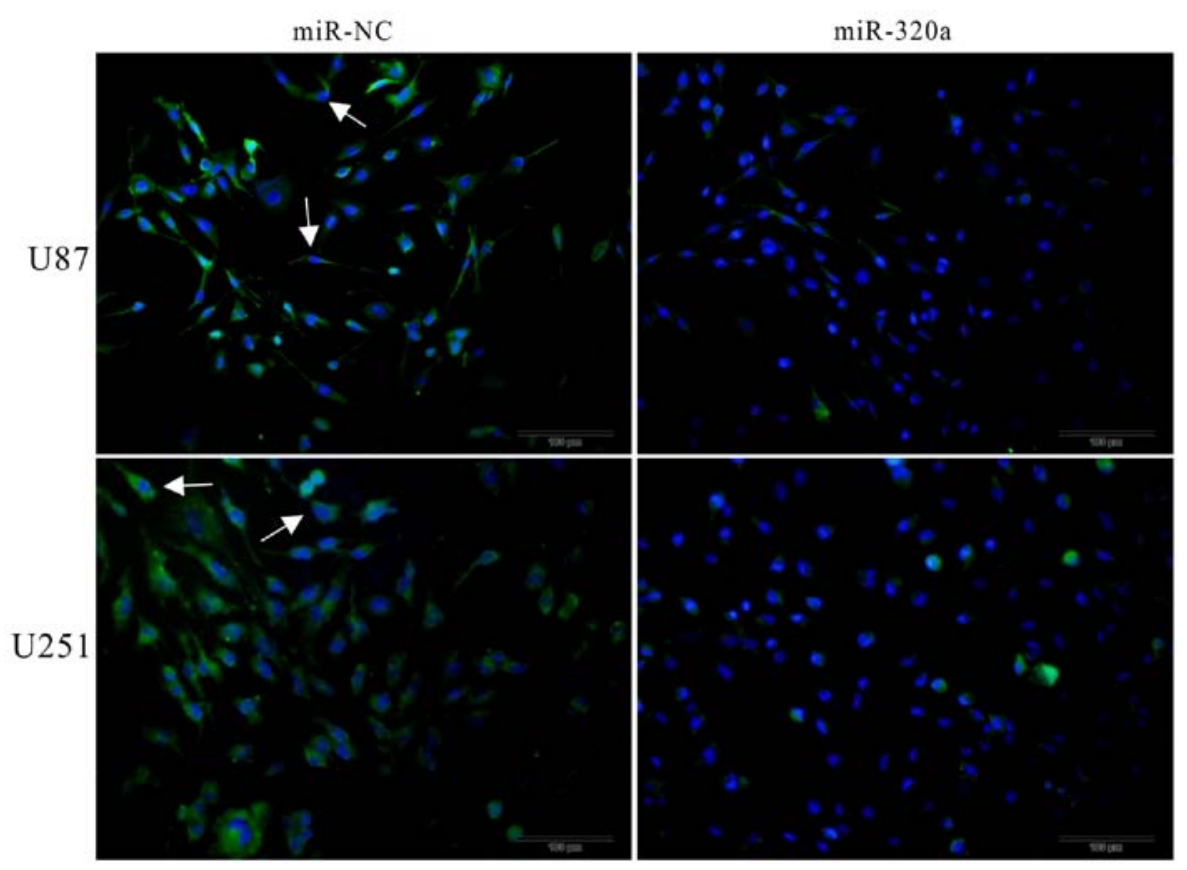

Figure 7. The expression and location of AQP4 was determined by immunofluorescence. The polarization of AQP4 in the miR-NC groups was marked by arrows. AQP4, aquaporin 4.
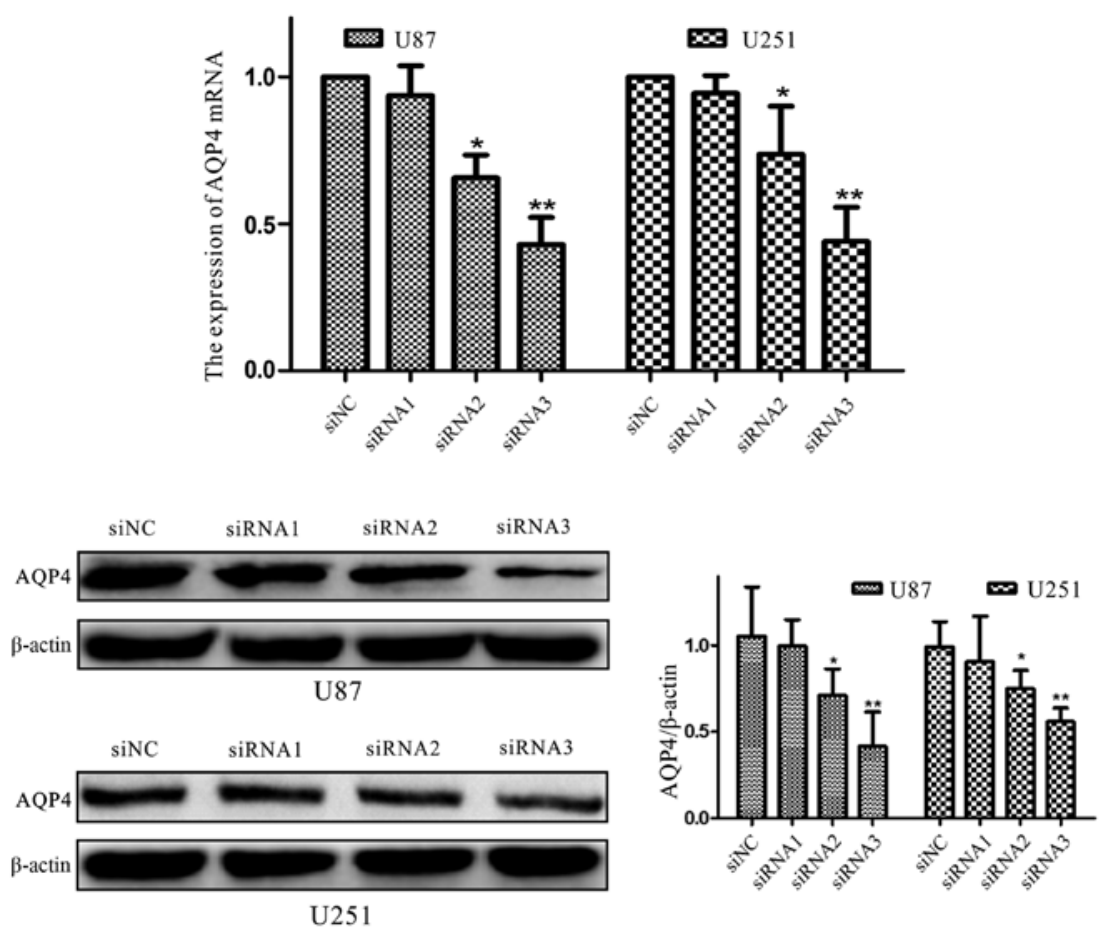

Figure 8. U87 and U251 cell lines were transfected with three independent interference fragments of AQP4, and RT-PCR and western blotting were used to filter out the most efficient interference fragment $\left({ }^{*} \mathrm{P}<0.05,{ }^{* *} \mathrm{P}<0.01\right)$. AQP4, aquaporin 4.

expression, and whether these changes could regulate the expression of the target protein AQP4, and MMP9. The results revealed that the mRNA and protein levels of AQP4 were significantly decreased however, MMP9 expression was not markedly altered (Fig. 6). Furthermore, the immunofluorescence assay revealed that AQP4 was mainly expressed on the cell membrane, that it had a polarized distribution, and that it was inhibited by the overexpression of miRNA-320a (Fig. 7).
These results indicated that miRNA-320a suppressed invasion and migration through direct interference with AQP4, and not with MMP9, in the U87 and U251 cell lines.

Interference with AQP4 expression inhibits cell invasion and migration. To further demonstrate the functions of AQP4 in U87 and U251 cells, we used RNA interference to downregulate the AQP4 protein $(\mathrm{P}<0.01$; Fig. 8). Immunofluorescence 
A
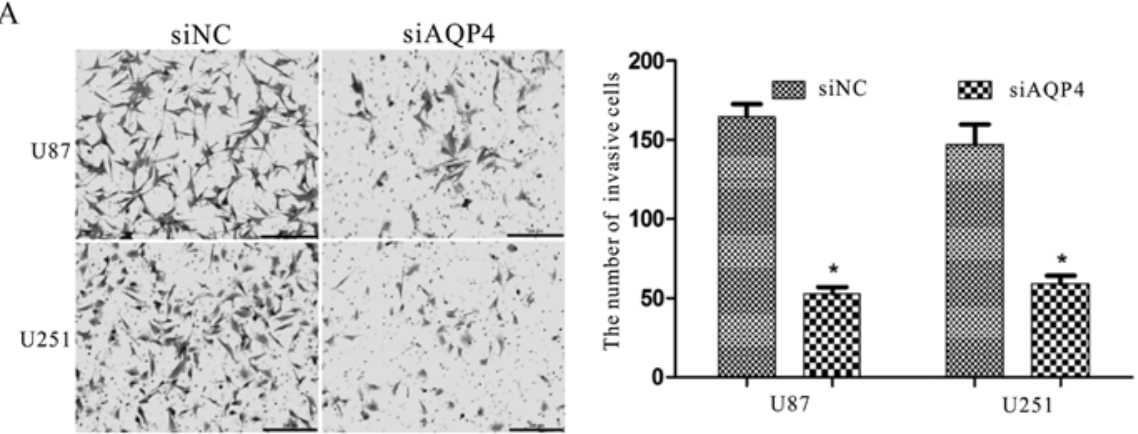

B
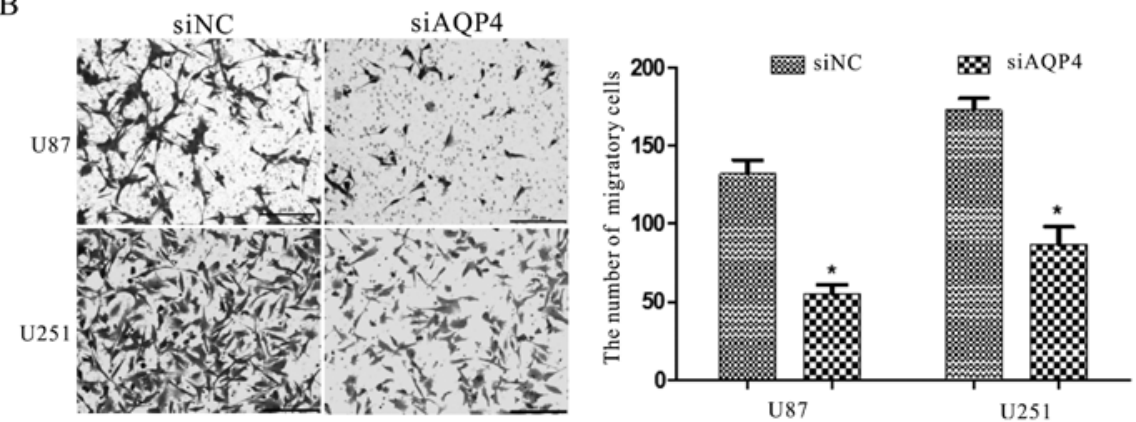

Figure 9. Cell invasion and migration were inhibited by AQP4 gene RNA interference. (A) The Transwell inner chamber was coated with matrix gel for cell invasion detection and cell counting. (B) The Transwell chamber without matrix gel directly determined the cell migration $\left({ }^{*} \mathrm{P}<0.05\right)$.

also was used to co-verify (Fig. 9) that the expression of AQP4 protein was significantly downregulated. The Transwell assay revealed that siAQP4 could markedly inhibit the invasion and migration of U87 and U251 cells (Fig. 9). These results demonstrated that AQP4 has a key function in glioma cell motility.

\section{Discussion}

An increasing number of studies have been focusing on the roles of various miRNAs in cancer. Many studies have revealed that miRNAs can serve key roles in regulating the expression of tumor suppressor genes or oncogenes, and that the aberrant expression of miRNAs could promote or inhibit oncogenesis $(4,10,12)$. Certain studies have stated that miRNAs regulate the self-renewal, differentiation and division of cells via post-transcriptional gene silencing (10). Aberrant miRNA levels, particularly an overall downregulation are present in various cancer types compared with their normal tissue counterparts. Thus, miRNAs are potential biomarkers for monitoring the progress and prognosis of patients with cancer.

Our results showed that miRNA-320a was significantly decreased in the glioma tissue samples analyzed with the CGGA microarray data. Combining the data with those of prior studies confirms that miRNA-320a is markedly lower in glioma tissues than in normal brain tissues, and lower in glioma cell lines than in normal human astrocytes (31). Thus, we hypothesized that the expression of miRNA-320a was aberrant in gliomas. As aforementioned, miRNA-320a has many targets. It can suppress the gene expression of IGF-1R, $\beta$-catenin, BCL/ABL and ARPP-19, among others $(13,29,31,35)$, acting as a tumor suppressor in these cancer types. Thus, miRNA-320a is significantly downregulated in gliomas and in other malignant tumor types, and miRNA-320a overexpression could

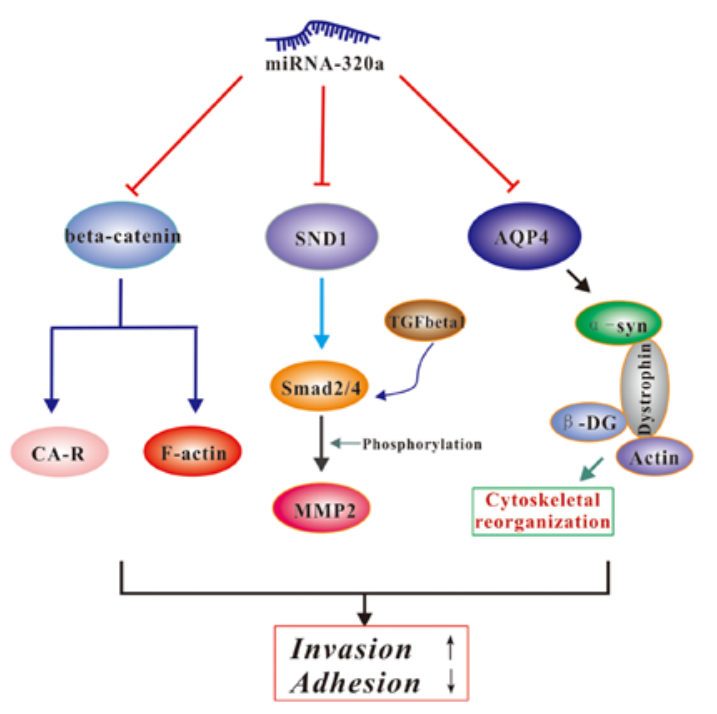

Figure 10. The roles of $\beta$-catenin, SND1 and AQP4 in glioma. SND1, staphylococcal nuclease domain-containing 1; AQP4, aquaporin 4; CA-R, cadherin adhesion receptor; $\beta$-DG, $\beta$-dystroglycan.

suppress tumor development in vivo and in vitro, potentially improving the prognosis.

The AQP family has thirteen members, of which AQP4 is the most studied; it has been described in the brain cells of both rodents and primates (19). The results of this particular study indicated that AQP4 is primarily permeable to water. In brain tissues, a high concentration of AQP4 has been documented at the astrocyte end-feet where they are in contact with blood vessels (19). However, AQP4 distribution differs significantly between various brain structures, including between astrocytes, the hippocampus, the cerebellum and the corpus 
callosum (36-40). The different AQP4 distribution patterns suggest that AQP4 has multiple functions in the brain. AQP4 may also be involved in cell adhesion (24). It has been reported in human breast cancer that the downregulation of AQP4 could inhibit cell proliferation, migration and invasion (33). In glioblastoma, AQP4 knockdown was revealed to induce cell apoptosis (23). In the present study, we determined that downregulation of AQP4 inhibited the invasion and migration of U87 and U251 glioma cells (Fig. 9). In autoimmune and neurodegenerative diseases, AQP4 has been associated with neuroinflammation $(41,42)$. Integration of this information revealed that AQP4 not only has a key role in cerebral fluid transportation, but also serves significant functions in pathological processes of the central nervous system. However, the potential functions of AQP4 in the central nervous system still need to be fully elucidated.

Matrix metalloproteinases (MMPs), which are secreted by cells, are able to degrade all components of the ECM, a process conducive to cell invasion and migration $(43,44)$. In particular, MMP9 has been demonstrated to be associated with numerous aggressive tumor types in humans, including glioma (45-47). In the present study MMP9 expression was not altered with the overexpression of miRNA-320a, thus confirming that miRNA-320a inhibits glioma cell invasion and migration by a mechanism other than targeting MMP9. However, we hypothesized that AQP4 and MMP9 may act synergistically in glioma cell invasion and migration to inhibit these processes.

A recent study found that miRNA-320a can directly target SND1 and $\beta$-catenin to inhibit glioma cell invasion and proliferation (32). Another study indicated that $\beta$-catenin could bind to cadherin adhesion receptors and F-actin in order to bridge the extracellular adhesive activity of cadherins with the actin cytoskeleton of tumor cells (48). Lan et al revealed that AQP4 was involved in cytoskeletal organization. Briefly, AQP4 interacts with $\alpha$-syntrophin; this is comprised of dystrophin and utrophin, linker molecules between the actin cytoskeleton and $\beta$-dystroglycan (22). SND1, also known as P100 co-activator or Tudor domain-containing protein 11, promotes tumor angiogenesis, cell invasion and cell migration via the $\mathrm{NF}-\kappa \mathrm{B}$ and TGF- $\beta$ signaling pathways $(49,50)$. Li et al reported that SND1 promotes Smad2/4 expression, a pivotal downstream signaling protein of SND1 in the TGF- $\beta 1$ pathway, and then enhances MMP2 expression (32). Collectively, the results indicated that AQP4, $\beta$-catenin and SND1 may all be regulated by miRNA-320a, that $\beta$-catenin and AQP4 could interact with adhesion-associated proteins and cytoskeletal proteins, and regulate the invasive ability of cells, and that SND1 promotes cell aggressiveness by degrading the ECM (Fig. 10).

In the present study, we identified AQP4 to be a direct and functional target of miRNA-320a. Overexpression of miRNA-320a in glioma cells suppressed cell invasion and migration. In addition, evaluation of the microarray data and survival analysis from the CGGA samples revealed that miRNA-320a was significantly downregulated in glioma tissues, which was correlated with prognosis. The present study demonstrated that miRNA-320a functions as a tumor suppressor in glioma, and that AQP4 serves an important function in cell invasion and migration. Although miRNA-based therapeutics are still under development, our results are encouraging, and suggest that miRNA-320a and AQP4 could serve as clinical targets for the treatment of glioma or other tumor types in the future.

\section{Acknowledgements}

The present study was supported by the National Science Foundation of China (grant no. 31271368). We thank Wei Qiang of The University of Chicago Molecular Oncology Laboratory for support with the theory.

\section{Competing interests}

The authors declare that they have no competing interests.

\section{References}

1. Vredenburgh JJ, Desjardins A, Reardon DA and Friedman HS: Experience with irinotecan for the treatment of malignant glioma. Neuro Oncol 11: 80-91, 2009.

2. Katakowski M, Charteris N, Chopp M and Khain E: Densitydependent regulation of glioma cell proliferation and invasion mediated by miR-9. Cancer Microenviron 9: 149-159, 2016.

3. Kim Y, Jeon H and Othmer H: The role of the tumor microenvironment in glioblastoma: A mathematical model. IEEE Trans Biomed Eng 64: 519-527, 2017.

4. Liz $\mathrm{J}$ and Esteller M: lncRNAs and microRNAs with a role in cancer development. Biochim Biophys Acta 1859: 169-176, 2016.

5. Zhang T, Wang YR, Zeng F, Cao HY, Zhou HD and Wang YJ: LncRNA H19 is overexpressed in glioma tissue, is negatively associated with patient survival, and promotes tumor growth through its derivative miR-675. Eur Rev Med Pharmacol Sci 20: 4891-4897, 2016

6. Oberheim Bush NA and Chang S: Treatment strategies for low-grade glioma in adults. J Oncol Pract 12: 1235-1241, 2016.

7. Weathers SS and Gilbert MR: Toward personalized targeted therapeutics: An overview. Neurotherapeutics 14: 256-264, 2017.

8. Bartel DP: MicroRNAs: Genomics, biogenesis, mechanism, and function. Cell 116: 281-297, 2004.

9. Miska EA: How microRNAs control cell division, differentiation and death. Curr Opin Genet Dev 15: 563-568, 2005.

10. Zimmerman AL and Wu S: MicroRNAs, cancer and cancer stem cells. Cancer Lett 300: 10-19, 2011.

11. Reid G: MicroRNAs in mesothelioma: From tumour suppressors and biomarkers to therapeutic targets. J Thorac Dis 7: 1031-1040, 2015.

12. Iorio MV and Croce CM: MicroRNAs in cancer: Small molecules with a huge impact. J Clin Oncol 27: 5848-5856, 2009.

13. Sun JY, Huang Y, Li JP, Zhang X, Wang L, Meng YL, Yan B, Bian YQ, Zhao J, Wang WZ, et al: MicroRNA-320a suppresses human colon cancer cell proliferation by directly targeting $\beta$-catenin. Biochem Biophys Res Commun 420: 787-792, 2012.

14. Mittal SP, Mathai J, Kulkarni AP, Pal JK and Chattopadhyay S: miR-320a regulates erythroid differentiation through MAR binding protein SMAR1. Int J Biochem Cell Biol 45: 2519-2529, 2013.

15. Shang C, Zhang H, Guo Y, Hong Y, Liu Y and Xue Y: miR-320a down-regulation mediates bladder carcinoma invasion by targeting ITGB3. Mol Biol Rep 41: 2521-2527, 2014.

16. Nagelhus EA and Ottersen OP: Physiological roles of aquaporin-4 in brain. Physiol Rev 93: 1543-1562, 2013.

17. Camassa LM, Lunde LK, Hoddevik EH, Stensland M, Boldt HB, De Souza GA, Ottersen OP and Amiry-Moghaddam M: Mechanisms underlying AQP4 accumulation in astrocyte endfeet. Glia 63: 2073-2091, 2015.

18. Hubbard JA, Hsu MS, Seldin MM and Binder DK: Expression of the astrocyte water channel aquaporin-4 in the mouse brain. ASN Neuro 7: 1759091415605486, 2015.

19. Badaut J, Fukuda AM, Jullienne A and Petry KG: Aquaporin and brain diseases. Biochim Biophys Acta 1840: 1554-1565, 2014.

20. Lan YL, Zhao J, Ma T and Li S: The potential roles of aquaporin 4 in Alzheimer's disease. Mol Neurobiol 53: 5300-5309, 2016.

21. Yang WC, Zhou LJ, Zhang R, Yue ZY, Dong H, Song CY, Qian H, Lu SJ and Chang FF: Effects of propofol and sevoflurane on aquaporin-4 and aquaporin- 9 expression in patients performed gliomas resection. Brain Res 1622: 1-6, 2015. 
22. Lan YL, Wang X, Lou JC, Ma XC and Zhang B: The potential roles of aquaporin 4 in malignant gliomas. Oncotarget 8: 32345-32355, 2017.

23. Ding T, Zhou Y, Sun K, Jiang W, Li W, Liu X, Tian C, Li Z, Ying $\mathrm{G}, \mathrm{Fu} \mathrm{L}$, et al: Knockdown a water channel protein, aquaporin-4, induced glioblastoma cell apoptosis. PLoS One 8: e66751, 2013.

24. Hiroaki Y, Tani K, Kamegawa A, Gyobu N, Nishikawa K, Suzuki H, Walz T, Sasaki S, Mitsuoka K, Kimura K, et al: Implications of the aquaporin-4 structure on array formation and cell adhesion. J Mol Biol 355: 628-639, 2006.

25. Yang L, Wang X, Zhen S, Zhang S, Kang D and Lin Z: Aquaporin-4 upregulated expression in glioma tissue is a reaction to glioma-associated edema induced by vascular endothelial growth factor. Oncol Rep 28: 1633-1638, 2012.

26. Zhao WJ, Zhang W, Li GL, Cui Y, Shi ZF and Yuan F: Differential expression of MMP-9 and AQP4 in human glioma samples. Folia Neuropathol 50: 176-186, 2012.

27. Peng J, Omran A, Ashhab MU, Kong H, Gan N, He F and Yin F: Expression patterns of miR-124, miR-134, miR-132, and miR-21 in an immature rat model and children with mesial temporal lobe epilepsy. J Mol Neurosci 50: 291-297, 2013.

28. Liu ZH, Li J, Xia J, Jiang R, Zuo GW, Li XP, Chen Y, Xiong W and Chen DL: Ginsenoside 20(s)-Rh2 as potent natural histone deacetylase inhibitors suppressing the growth of human leukemia cells. Chem Biol Interact 242: 227-234, 2015.

29. Xishan Z, Ziying L, Jing D and Gang L: MicroRNA-320a acts as a tumor suppressor by targeting BCR/ABL oncogene in chronic myeloid leukemia. Sci Rep 5: 12460, 2015.

30. Zhang G, Jiang G, Wang C, Zhong K, Zhang J, Xue Q, Li X, Jin H and Li B: Decreased expression of microRNA-320a promotes proliferation and invasion of non-small cell lung cancer cells by increasing VDAC1 expression. Oncotarget 7: 49470-49480, 2016.

31. Guo T, Feng Y, Liu Q, Yang X, Jiang T, Chen Y and Zhang Q: MicroRNA-320a suppresses in GBM patients and modulates glioma cell functions by targeting IGF-1R. Tumour Biol 35: 11269-11275, 2014.

32. Li H, Yu L, Liu J, Bian X, Shi C, Sun C, Zhou X, Wen Y, Hua D, Zhao $\mathrm{S}$, et al: miR-320a functions as a suppressor for gliomas by targeting SND1 and $\beta$-catenin, and predicts the prognosis of patients. Oncotarget 8: 19723-19737, 2017.

33. Li YB, Sun SR and Han XH: Down-regulation of AQP4 inhibits proliferation, migration and invasion of human breast cancer cells. Folia Biol (Praha) 62: 131-137, 2016.

34. Cheng C, Chen ZQ and Shi XT: MicroRNA-320 inhibits osteosarcoma cells proliferation by directly targeting fatty acid synthase. Tumour Biol 35: 4177-4183, 2014.

35. Lü M, Ding K, Zhang G, Yin M, Yao G, Tian H, Lian J, Liu L, Liang M, Zhu T, et al: MicroRNA-320a sensitizes tamoxifen-resistant breast cancer cells to tamoxifen by targeting ARPP-19 and ERR $\gamma$. Sci Rep 5: 8735, 2015.

36. Badaut J, Nehlig A, Verbavatz J, Stoeckel M, Freund-Mercier MJ and Lasbennes F: Hypervascularization in the magnocellular nuclei of the rat hypothalamus: Relationship with the distribution of aquaporin- 4 and markers of energy metabolism. $\mathrm{J}$ Neuroendocrinol 12: 960-969, 2000.

37. Badaut J, Verbavatz JM, Freund-Mercier MJ and Lasbennes F: Presence of aquaporin- 4 and muscarinic receptors in astrocytes and ependymal cells in rat brain: A clue to a common function? Neurosci Lett 292: 75-78, 2000.
38. Hsu MS, Seldin M, Lee DJ, Seifert G, Steinhäuser C and Binder DK: Laminar-specific and developmental expression of aquaporin-4 in the mouse hippocampus. Neuroscience 178: 21-32, 2011.

39. Nielsen S, Nagelhus EA, Amiry-Moghaddam M, Bourque C, Agre P and Ottersen OP: Specialized membrane domains for water transport in glial cells: High-resolution immunogold cytochemistry of aquaporin-4 in rat brain. J Neurosci 17: 171-180, 1997.

40. Wen H, Nagelhus EA, Amiry-Moghaddam M, Agre P, Ottersen OP and Nielsen S: Ontogeny of water transport in rat brain: Postnatal expression of the aquaporin-4 water channel. Eur J Neurosci 11: 935-945, 1999.

41. Juryńczyk M, Tackley G, Kong Y, Geraldes R, Matthews L, Woodhall M, Waters P, Kuker W, Craner M, Weir A, et al: Brain lesion distribution criteria distinguish MS from AQP4-antibody NMOSD and MOG-antibody disease. J Neurol Neurosurg Psychiatry 88: 132-136, 2017.

42. Vaknin-Dembinsky A, Brill L, Kassis I, Petrou P, Ovadia H, Ben-Hur T, Abramsky $\mathrm{O}$ and Karussis D: T-cell responses to distinct AQP4 peptides in patients with neuromyelitis optica (NMO). Mult Scler Relat Disord 6: 28-36, 2016.

43. Roomi MW, Monterrey JC, Kalinovsky T, Rath M and Niedzwiecki A: Inhibition of invasion and MMPs by a nutrient mixture in human cancer cell lines: A correlation study. Exp Oncol 32: 243-248, 2010.

44. Singh RD, Haridas N, Patel JB, Shah FD, Shukla SN, Shah PM and Patel PS: Matrix metalloproteinases and their inhibitors: Correlation with invasion and metastasis in oral cancer. Indian J Clin Biochem 25: 250-259, 2010.

45. Das G, Shiras A, Shanmuganandam K and Shastry P: Rictor regulates MMP-9 activity and invasion through Raf-1-MEK-ERK signaling pathway in glioma cells. Mol Carcinog 50: 412-423, 2011.

46. Ruiz-Morales JM,Dorantes-HerediaR,Arrieta O,Chávez-Tapia NC and Motola-Kuba D: Neutrophil gelatinase-associated lipocalin (NGAL) and matrix metalloproteinase-9 (MMP-9) prognostic value in lung adenocarcinoma. Tumour Biol 36: 3601-3610, 2015.

47. Shi W, Xiao H, Xue F and Wu J: Dynamic changes of matrix metalloproteinase 9 in heterotopic ossification of rat model. Zhongguo Xiu Fu Chong Jian Wai Ke Za Zhi 28: 1133-1138, 2014 (In Chinese).

48. Rimm DL, Koslov ER, Kebriaei P, Cianci CD and Morrow JS: Alpha 1(E)-catenin is an actin-binding and -bundling protein mediating the attachment of F-actin to the membrane adhesion complex. Proc Natl Acad Sci U S A 92: 8813-8817, 1995.

49. Santhekadur PK, Akiel M, Emdad L, Gredler R, Srivastava J, Rajasekaran D, Robertson CL, Mukhopadhyay ND, Fisher PB and Sarkar D: Staphylococcal nuclease domain containing-1 (SND1) promotes migration and invasion via angiotensin II type 1 receptor (AT1R) and TGF $\beta$ signaling. FEBS Open Bio 4: 353-361, 2014.

50. Santhekadur PK, Das SK, Gredler R, Chen D, Srivastava J, Robertson C, Baldwin AS Jr, Fisher PB and Sarkar D: Multifunction protein staphylococcal nuclease domain containing 1 (SND1) promotes tumor angiogenesis in human hepatocellular carcinoma through novel pathway that involves nuclear factor $\kappa \mathrm{B}$ and miR-221. J Biol Chem 287: 13952-13958, 2012. 